\title{
A Framework for BPM Software Selection in Relation to Digital Transformation Drivers
}

\author{
Ljiljana BRKIĆ, Katarina TOMIČIĆ PUPEK, Vesna BOSILJ VUKŠIĆ
}

\begin{abstract}
Business process management (BPM) is nowadays almost a traditional paradigm of assuring operational excellence. Within emerging approaches for boosting organizational readiness and maturity to cope with digital challenges, BPM transforms as well. In order to investigate the role of BPM and BPM software (BPMS) in relation to these approaches we explore drivers of digital transformation (DT). Based on the mapping of BPMS dimensions and drivers of DT, we propose a framework for selfassessment which allows evaluation of the significance of BPMS dimension in relation to DT. The framework was tested on multiple case-studies to analyse what their BPMS priorities are and how these priorities influence their digital transformation. AHP analysis was performed by prioritizing BPM's dimensions mapped with DT drivers and it served as a basis for assessing the level of digital maturity of the observed companies. This article therefore deals with the following: (1) it briefly discusses BPM and DT as concepts, (2) it demonstrates how AHP can serve for selecting BPM software and (3) it shows that BPM dimensions can be linked to Digital Transformation.
\end{abstract}

Keywords: AHP; business process management software; digital transformation drivers

\section{INTRODUCTION}

Drivers of Digital Transformation can be found in a variety of sources and numerous academic and scientific articles. For a successful digital transformation, it is crucial to choose a right strategy and appropriate technologies, while keeping in mind the existing limitations. Designing new business models based on customer expectations (existing or planned) includes a) mastering touch points design with great complexity of interactions across multiple channels as well as b) adapting the organization in such a way that it can deliver on its value proposition [2]. This includes the design of new business models and a serious business process reinvention, resource management, capacity building and other organizational and technological empowering possibilities.

The impression that Digital Transformation is an inevitable challenge, as well as a great opportunity, arises from the impact projections that "current, emerging, and disruptive technology has had on business processes and whole new business models" [3]. Knowledge, skills, experience and vendor support for modelling, simulation of existing and future performance, and continuous management of business processes are the "must haves" for implementing flexible and adaptive business processes.

Process flexibility can be described as ability to orchestrate behaviour and business logic while reacting to disruptions or acting on data and information gathered from interconnected (e.g. IoT) devices. BPM Software can be seen as a tool which enables the implementation of flexible and adaptive business processes, which on the other hand allows us to treat these software systems as a special kind of self-adaptive systems [4]. In order to explore the role of BPM Software in the context of DT we have set the following objectives:

(O1) investigate the role of BPM software in relation to DT's drivers i.e. explore the connection between the technological dimensions of BPMS and DT drivers,

(O2) propose a framework for self-assessment by mapping technological BPMS dimensions with DT drivers (shorter FSADT) for evaluating the significance of BPMS dimension in relation to DT,

(O3) test the framework for self-assessment on multiple case-studies to analyse what their priorities are in terms of
BPM software features and to see if these priorities lead in the direction of digital transformation.

To achieve the objectives, we first create a concept centric review of DT drivers, then form our framework by mapping technological dimensions of BPMS to these drivers. For the implementation of our framework we rely on the use of AHP method which should allow us to test the framework on 3 case studies of organizations which participated in a survey on BPMS. In the final part of our paper, we synthetize on validity of the framework, examine implications for practice and comment on limitations and future research directions.

\section{RESEARCH METHODOLOGY \\ 2.1 Framework Design}

The research framework was developed through the analysis of literature found by searching for a key word "driver" in professional digital transformation reports and most relevant digital business model templates which are available as publications on platforms ran by leading consulting organizations like Mc Kinsey \& Company [5], TM Forum [6], Forbes [7], Forrester [8] and others. IT professionals and the academia are showing a similar standpoint regarding the key enabling means and drivers: digital technologies can be seen as one of leading drivers, but they cannot stand alone. According to Von Leipzig et al. [9] digital maturity models include more than just technology-related concepts. By implementing a snowball approach in literature research based on references in the publication of von Leipzig et al. [9], other relevant publications were gathered. All of them showed that digital transformation is more about the business change itself, whereby digital technologies are one of most important enablers. Four drivers of digital transformation recognized in the literature are systemized in Tab. 1. The main concepts of drivers from the perspective of professionals, academics and researchers are listed and the sources are cited.

Technologies implemented in digital transformation initiatives are also known as technologies of the fourth industrial revolution (Industry 4.0) and include Internet of Things (IoT), Cyber Physical System (CPS), Enterprise Architecture (EA), Enterprise Integration (EI) [10], smart 
materials, mobile and autonomous systems, virtual and augmented reality and other [11]. Technology alone cannot deliver the expected change. In order to accelerate the digital business, companies have to incorporate change into their operating models and effectively execute digital initiatives [8]. Digital transformation initiatives can be initiated by disruptive competitors or by digitally matured customers whose expectations and experience in acquiring products or services forces companies to go digital. Newman states that the digital change is only possible if digital initiative is built in the right kind of organization that embraces what is possible in terms of customer experience, people, change, innovation, leadership, and culture [7]. Customer value proposition [12] and Business model Canvas [2] are two well-known frameworks for building awareness about customer centricity in DT and the need for redesigning organizational business operating models. Digitally operating business models combine "digital technologies and operations capabilities in an integrated, well-sequenced way to achieve step-change improvements in revenue, customer experience, and cost" [5].

Table 1 Drivers of DT with description and sources

\begin{tabular}{|l|l|l|}
\hline \multicolumn{1}{|c|}{$\begin{array}{c}\text { Drivers of Digital } \\
\text { Transformation }\end{array}$} & \multicolumn{1}{|c|}{ Concepts linked to driver } & \multicolumn{1}{|c|}{ Source } \\
\hline Technology-oriented DT & $\begin{array}{l}\text { Digital technologies or Industry 4.0. technologies: IoT, Big } \\
\text { Data, Data analytics, Mobile technologies, Cloud } \\
\text { technologies, virtual and augmented reality, robotics, } \\
\text { autonomous systems, chatbots, blockchain, smart/wearable } \\
\text { materials, machine learning; }\end{array}$ & $\begin{array}{l}\text { Digital Maturity model 5.0. [8] } \\
\text { Technology and Capabilities [13] } \\
\text { Digital Technologies [11] } \\
\text { Industry 4.0. technologies [10] }\end{array}$ \\
\hline Customer-driven DT & $\begin{array}{l}\text { Customer journey, customer mapping, social media and } \\
\text { platforms, customer profiling, new value creation; }\end{array}$ & $\begin{array}{l}\text { Customer orientation [6] } \\
\text { Experiences [7] } \\
\text { Customer value proposition [12] } \\
\text { Customer experience [2] }\end{array}$ \\
\hline Governance-led DT & $\begin{array}{l}\text { Strategy, mission, vision, scope, cascading through the } \\
\text { organizational channels, governance and operating business } \\
\text { rules, supply chain in the context of ecosystems, } \\
\text { competitors in the context of ecosystems, circular economy, } \\
\text { organizational culture; }\end{array}$ & $\begin{array}{l}\text { Scope [14] } \\
\text { Strategy and vision, Process and governance, People and } \\
\text { Culture [13] }\end{array}$ \\
\hline Culture, Leadership [7] \\
\hline Capability-based DT & $\begin{array}{l}\text { Resource management, Innovation capacity, internal } \\
\text { process capacity, maturity assessment, integration } \\
\text { capability, simplicity, complexity, sustainability, internal } \\
\text { efficiency, improvement goals related to quality, time to } \\
\text { market, product/ service flexibility; }\end{array}$ & $\begin{array}{l}\text { Technology and Capabilities [13] } \\
\text { Digital Maturity Assessment Tool Government of South } \\
\text { Australia [15] } \\
\text { Change, Innovation, People [7] } \\
\text { Capabilities to drive the next-generation operating model [5] }\end{array}$ \\
\hline
\end{tabular}

Table 2 Technological dimensions mapped to DT drivers

CSFs of BPMS adoption [16]

CAP-1: Process modelling, analysis and design: capability to identify, analyse and propose improvements

CAP-2: Business rules: understanding, innovating and implementing business rules to increase internal efficiency

CAP-3: Reporting, analytics, monitoring: a significant element in exploiting technologies, like data analytics in the context of DT

CAP-4: Social BPM: Connection to Social BPM, Customer journey and Customer Experience Management as one of key motivators of Digital Change

CAP-5: Process strategy subsystem: strategy operationalization by cascading responsibilities for process activities

CAP-6: Low-code development: ability to interconnect process-oriented software with industry 4.0. technologies (e.g. robotics, autonomous subsystems, sensors), integration, service-oriented architecture

CAP-7: Enactable models and process engine: transform process models into process-oriented software, implementation of the model-driven-development paradigm

CAP-8: Mobile \& tablet functionalities: mobile technologies as one of key technologies in industry 4.0. and DT, 24/7 availability of BPMS for process actors, actor-location variability

CAP-9: Web platform, cloud capabilities: 24/7 availability of services, process-location variability

CAP-10: Security and reliability: going-digital raises demands on security of cloud and mobile based BPMS solutions, reinventing communication channels, rethinking reliability options

COMPA-1: Existence of compatibility: digital technology implementation with high-level of compatibility to professional standards, legacy software, new customer requirements, needs and beliefs

COMPA-2: Simplicity of integration: easiness of integration by following SaaS paradigm, capability of maintaining serviceoriented architecture

COMPL-1: BPMS implementation complexity: overall impression about the complexity of the initiative, technology vs. user centricity, service flexibility

COMPL-2: Simplicity of BPMS use: simplicity of BPMS implementation in relation to the level of skills required, human resource management

COMPL-3: BPMS user interface complexity: dedication to shortening the learning curve, easiness of use on various platforms REP-1: Vendor maturity: vendor's knowledge, references, experience, service availability

REP-2: Presence on the local market: dedication to local values, building ecosystems with vendors for assuring long-term commitment and sustainability

REP-3: BPMS documentation: level of simplicity to generate useful documentation for next-generation operating models

REP-4: BPMS installation and maintenance: maintenance availability as an important aspect of vendors service availability, raising complexity of requirements and functionalities

COST-1: BPMS implementation costs in relation to budget: cost structure and organizational commitment to change, gains of the initiative 
Tab. 2 shows eighteen technological critical success factors (CSFs) for BPMS adoption according to the findings of Bosilj Vukšić et al. [16]. The CSFs are sorted in five categories and are mapped according to the results of the literature review of DT drivers conducted for the purpose of this study.

Questionnaire used for the survey on investigating CSFs for BPMS adoption [16] had both contextual and technological dimensions. Contextual dimensions were Organizational (ORG) and Environmental (ENV), and technological dimensions addressed BPMS capability (CAP), BPMS compatibility (COMPA), BPMS complexity (COMPL), BPMS vendor's reputation and maturity (REP) and BPMS implementation costs and benefits (COST). Each technological dimension had one or more elements which referred to the functionalities of BPMS. The elements describing various aspects of the technological dimension of BPMSs are mapped across drivers of Digital Transformation (Tab. 2).

The findings presented in Tab. 1 and Tab. 2 refer to the first two research objectives:1) the investigation of the role of BPM software in relation to DT's drivers and 2) designing the mapping structure for the self-assessment framework. These findings were then used for testing the framework for self-assessment on three case-studies from organizations which participated in a multiple case study analysis on BPMS CFS. This approach can be considered suitable for examining real-life experiences in terms of BPM software features and in rethinking priorities which are relevant in the context of digital transformation.

\subsection{Development of Framework and Data Collection}

A multiple case study approach is used to test the proposed framework. Yin [17] noted that case study information may come from many sources. Besides direct observations, the interview is one of the most important ones. We used a semi-structured interview to gather both quantitative data for topics that can be evaluated numerically, and qualitative contextual (organizational and environmental) data relevant for BPMS adoption success.

The questionnaire had two parts: (i) one where interviewees answered the questions in a free-form style to describe contextual CSFs and (ii) the other where interviewees evaluated technological CSFs by means of the Likert scale. For each statement from the second part of the questionnaire the interviewees were asked to give an importance score on the scale from 1 to 5 , with the meaning: 1 = "not important at all"; $2=$ "of little importance"; 3 = "of average importance"; 4 = "very important"; 5 = "absolutely essential". The option "X = don't know" was also available. The study includes three organizations that have different ownership, size, type of business and process characteristics. In the rest of the paper, for the protection of identity and confidential information, we use generic names for organizations: "Organization A", "Organization B" and "Organization C".

The quantitative, numerical data collected are processed using the multi criteria decision making methodthe Analytic Hierarchy Process (AHP). AHP is a structured technique that helps decision makers find solution that suits their goal best. Because of its well-known advantages, this method has been used successfully in various fields.
Reviewing the literature on this topic we found three types of problems that are solved by using AHP: (i) selection of most suitable alternative, (ii) ranking the alternatives, and (iii) ranking the indicators.

Castañon Guimarães, Leal and Mendes [18] use AHP to support software selection by weighting the components important for the specific company. In [19] Veronese Bentes, Carneiro, Ferreira da Silva and Kimura described usage of AHP in prioritizing multiple performance perspectives and indicators and in generating a unified metric for the ranking of alternatives.

When applying AHP, the first step is to identify elements describing any aspect of the decision problem and organize them in a hierarchy. Once the hierarchy is determined, elements are compared to one another with respect to their impact on an element above them in the hierarchy. The result of such comparison is ultimately integer, but in obtaining that number the decision makers typically rely on their understanding and knowledge about the elements' relative importance. In this process, Saaty's fundamental scale of relative importance given in Fig. 1, with the range of values from 1 to 9 , is used [20].

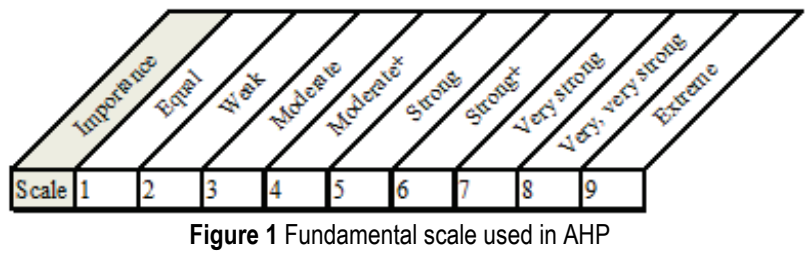

When, for example, in pairwise comparison of two elements $\mathrm{A}$ and $\mathrm{B}$, relative importance is set to 7 , that means that contribution to the objective of element $A$ is favoured very strongly over the contribution of element B.

Besides the underlying data, human common sense is used in evaluations. Therefore, AHP is said to be based on mathematics and psychology. The advantage of using numerical weight or priority for each element of the hierarchy is the ability of performing a consistent numerical procedure in their processing. In the final step of the process, numerical priorities are calculated for each of the decision alternatives. These numbers represent the alternatives' relative ability to achieve the decision goal, hence they allow a straightforward consideration of the various courses of action.

According to the review reported in [21], the hierarchical structure in 33 cases observed has one to three levels. The dominant is the two-level structure. The first level of a hierarchical structure consists of an objective or a goal that needs to be achieved. In the second level of indicators, a minimum of 2 and a maximum of 20 indicators were observed.

\subsubsection{Using AHP to Prioritize CSFs of BPM Adoption and Digital Transformation Drivers}

In this research we use AHP not to find the best solution and make a decision, but to benefit from the mathematical procedure that exists at the core of AHP method and is used to establish priorities among a number of different indicators. Specifically, the AHP method was used to (i) determine priorities between technological categories of BPMS adoption and (ii) determine whether 
these priorities lead in the direction of digital transformation.

In both cases we have one-level hierarchy structure. In the first case 20 and in second 4 indicators to rank. When prioritizing technological categories of BPMS, 20 indicators correspond to 20 categories (CAP-1 to COST-1) out of 5 technological dimensions (CAP, COMPA, COMPL, REP and COST). These 20 categories were numerically evaluated for each organization participating in the study by two types of participants: BPM practitioner and BPM expert. The BPM practitioners are employees involved in BPMS adoption and their opinion is particularly important for accomplishing the goal of this research-to verify the framework for self-assessment of the organization in the context of BPM software's priorities. Experts' opinion is not relevant in this research because they do not have to be (and in our cases they are not) employees of a company where the BPM project is implemented.

In the second case, values for digital transformation's drivers were computed as average values of grades given to corresponding technological categories of BPMS.

\begin{tabular}{|c|c|}
\hline \multicolumn{2}{|c|}{ Algorithm 1: Converting grades to Saaty's scale } \\
\hline 1 & Input: grades $\mathrm{Gi}$ and $\mathrm{Gj}$; \\
\hline 2 & Output: weight wij from saaty's scale; \\
\hline 3 & Begin \\
\hline 4 & 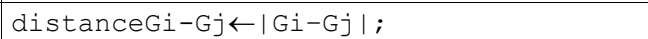 \\
\hline \multirow[t]{2}{*}{5} & if distanceGi-Gj $=0 \mathrm{wij}=0$ \\
\hline & wij $=1 ;$ \\
\hline 6 & else if distanceGi-Gje $(0,0.5]$ \\
\hline 7 & If $\mathrm{Gi}>\mathrm{Gj}$ then \\
\hline 8 & wij $=2$; \\
\hline 9 & Else \\
\hline 10 & $w i j=1 / 2 ;$ \\
\hline 11 & else if distanceGi-Gje $(0.5,1]$ \\
\hline 12 & If Gi>Gj then \\
\hline 13 & wij $=3$; \\
\hline 14 & Else \\
\hline \multirow[t]{2}{*}{15} & $w i j=1 / 3 ;$ \\
\hline & $\ldots$ \\
\hline 16 & else if distanceGi-Gje $(3.5,4]$ \\
\hline 17 & If $G i>G j$ then \\
\hline 18 & wij $=9$; \\
\hline 19 & Else \\
\hline 20 & $w i j=1 / 9 ;$ \\
\hline 21 & End \\
\hline
\end{tabular}

To be able to assess relative importance of one indicator compared to others, we must present all possible combinations of indicator's values with numerics from Saaty's scale. In the first case we have integers (1 - 5) since our interviewees used Likert scale and decimals in the second case. We used simple principle described with pseudo code named Algorithm 1. For two grades Gi and $\mathrm{Gj}$, a distance is calculated (line 4). The minimal distance is 0 and it appears for the grades having the same value. In this case assigned weight wij is 1 meaning both indicators contribute equally. When the distance is between 0 and 0.5 (line 6) assigned weight is 2 in case of comparison "higher grade-lower grade" or $1 / 2$ for the comparison "lower gradehigher grade". The algorithm continues further following this principle - increasing the weight by one each time the distance is incremented by 0.5 . The maximal weight 9 or
$1 / 9$ (line 18 and 20) is assigned for grades with the distance between 3.5 and 4 (line 16).

When prioritizing technological categories of BPMS we have $20 \times 20=400$ pairwise comparisons per each organization, 1200 estimates in total for three organizations. It would not be feasible without a welldefined mathematical procedure like the one used in AHP method.

\subsubsection{Basic Contextual Data about Organizations and BPM Initiatives Included in the Research}

The first organization, "Organization A", comes from the Croatian public sector. It grants the concessions to Croatian coastal liners and enables discount prices for the residents of Croatian islands. The first BPM project was started in 2014. It lasted one year and was not followed by the next project till nowadays. Bizagi BPM Suite was used to develop a new business application that supports the selected process. Additionally, several software tools and platforms were used, such as: Visual Paradigm for UML, SQL Server 2012, .NET Framework 4.4, ASP.NET MVC and WCF.

The second organization, "Organization B", is a largesized company from Bosnia and Herzegovina with more than 1.000 employees in telecommunications business. The first BPM initiative started in 2016 resulted in the successfully implemented BPMS which supports 3 business processes. Oracle Business Process Management Suite $12 \mathrm{c}$ was used for BPMS development.

The third organization, "Organization C", is a Croatian international airport with about 370 employees and with almost 3 million passengers in 2017. The first BPM initiative began in 2002 and was followed by many others, covering all of the phases of BPM lifecycle. Software AG (previously named ARIS) is implemented and used as a basic software platform for the BPMS development.

Several authors [16, 22, 23] identified five stages through which the BPM adoption typically goes: (1) awareness and understanding of BPM; (2) desire to adopt BPM; (3) BPM project; (4) BPM program; (5) productisation of BPM. According to the above stated regarding the BPM initiatives, Organization A can be assumed to be at the third stage of BPM adoption, Organization B in the fourth stage and Organization $\mathrm{C}$ in the final-fifth stage of BPM adoption.

\section{RESULTS OF AHP CALCULATIONS AND DISCUSSION}

Fig. 1 presents the result of prioritizing technological categories of BPMS in three organizations from practitioners' perspective after BPMS adoption. The analysis of the results aims to show their contribution to the fulfilment of the third research objective (O3).

The value on vertical axe is percentage of importance belonging to particular technological category. From the graph we can see that there are categories with harmonized opinions across organizations (e.g. CAP-1, CAP-2, CAP10, COMPA-1, COMPA-2, REP-2, REP-2 and REP-4), as well as categories where that is not the case (e.g. CAP-3, CAP-5, CAP-6, CAP-7, CAP-9, etc). Discrepancy in priorities is understandable given the variety of organizations' stage of BPM adoption. It is acceptable that 
the organization in the third stage of BPM adoption has different priorities of the technological categories than the organization that is at the most advanced stage.

Regardless of the BPM adoption stage, for some technological dimensions the opinion of all practitioners coincides i.e. they are the best ranked or the worst ranked. Top ranked dimensions by all the participants are:

- Process modelling, analysis and design (CAP-1),

- Business rules modelling (CAP-2),
- Security and reliability (CAP-10),

- Existence of compatibility (COMPA-1),

- Simplicity of integration (COMPA-2),

- Presence on a local market (REP-2),

- BPMS documentation (REP-3),

- $\quad$ BPMS installation and maintenance (REP-4).

Since behind each dimension stand functionalities and features of BPMSs, functionalities related to above listed dimensions can be considered as the core one

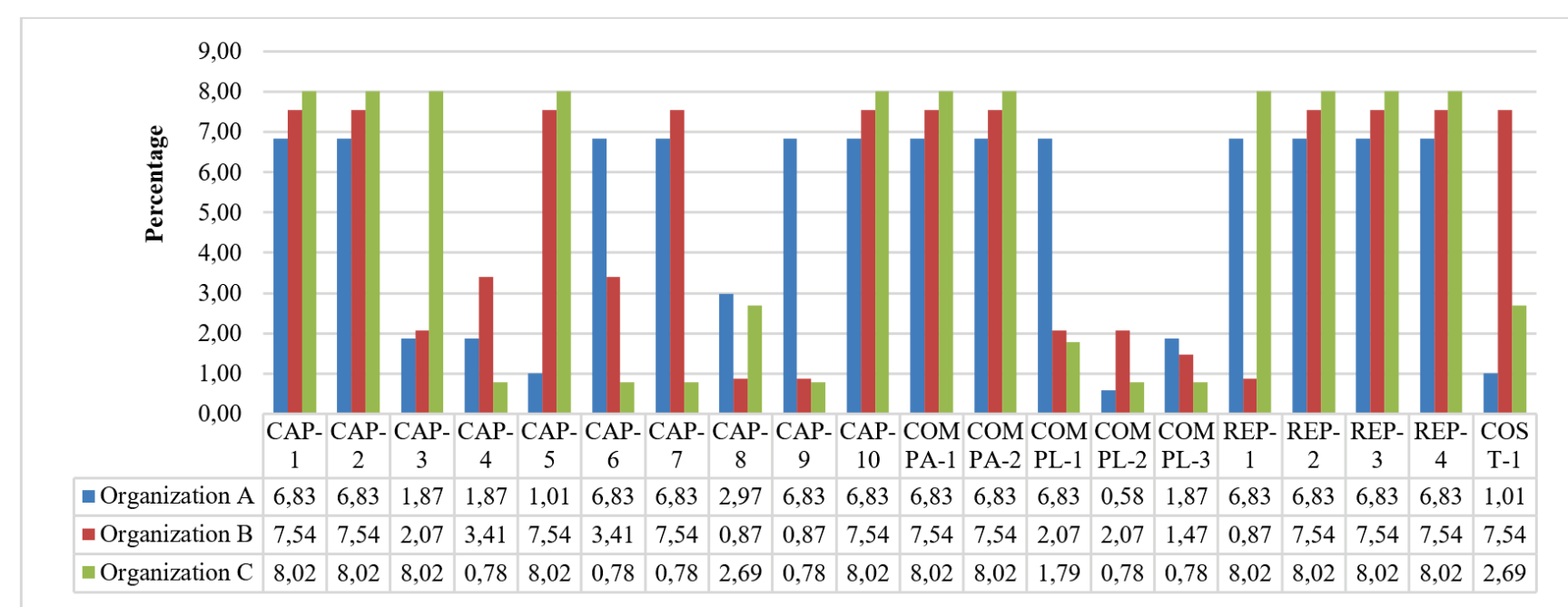

Figure 2 Technological categories of BPMS-prioritized with AHP by BPM practitioners after BPMS adoption

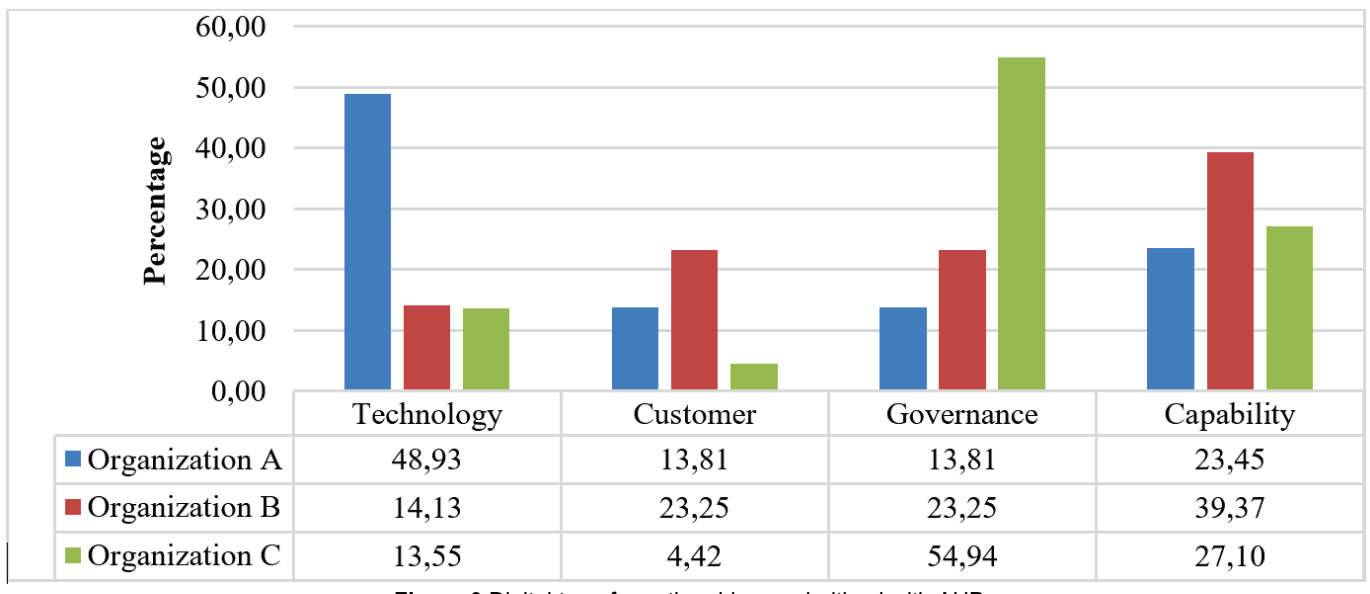

Figure 3 Digital transformation drivers prioritized with AHP

The worst ranked dimensions are:

- Social BPM (CAP-4),

- Mobile \& tablet functionalities (CAP-8),

- BPMS implementation complexity (COMPL-1),

- Simplicity of BPMS use (COMPL-2).

Social BPM as well as mobile \& tablet functionalities can be assumed as advanced BPMS's functionalities and are not required with every type of business process. The reason why the last two dimensions are poorly ranked may be one of the following two: (i) the knowledge and experience of a practitioners is very high and the complexity of BPMS does not represent a challenge or (ii) BPMS adoption is carried out by outsourcing external experts, and organizations are not aware of the problems they would face when implementing BPMS themselves.

It is also evident that there are different attitudes about some dimensions between study participants. Discrepancies are most evident on issues about reporting, analytics, monitoring (CAP-3), web platform, cloud capabilities (CAP-9), BPMS implementation complexity (COMPL-1), Vendor's maturity (REP-1) and BPMS implementation costs related to a budget (COST-1). It is interesting to note that the dimension related to reporting, analytics and monitoring is of the highest importance to the participants in the most advanced BPM adoption stage, while to participants on the lower stage they are not so important. Besides different stages of BPM adoption in the organization, some reasons that can justify these divergences are differences that exist in (i) organizational and environmental factors, (ii) types of processes implemented, (iii) final phases of implementation for particular process.

After applying the marks given by participants of our study in testing the framework for self-assessment, we can analyse what their priorities were in terms of evaluating BPM software features (Fig. 3). This allows us to 
investigate if and how these priorities lead in the direction of digital transformation. The testing was performed by calculating values for digital transformation's drivers which were computed as average values of grades given to corresponding technological categories of BPMS.

Most digital technologies have been developed independently of one another and independently from the trend of digital transformation, but they can all be used simultaneously to support organizational DT [24]. Moving away from merely transaction-oriented systems is crucial for maturing in the digital era. Systems of engaging collaboration, communication and cooperation should be more agile than systems of records whose main purpose is to support transactional activities [25]. The agility is not just the result of implementing digital technologies; it is the result of transforming the operational business model in a broader scope. Therefore, many maturity models implement a holistic approach in evaluating the level of digital transformation and maturity. Industry 4.0-Maturity model (MM) e.g. consists of the assessment of process transformation, application management, data governance, asset management, and organizational alignment areas and evaluates maturity on the scale from level 0: Incomplete to level 5: Optimizing [26]. Another often referenced digital maturity model (DMM) comes from the Reutlingen University and associates from a consulting company which takes into account 8 dimensions (Strategy, Leadership, Products, Operations, Culture, People, Governance and Technology) to evaluate the level of digital maturity from Unaware to Transformed [27].

Organization A evaluated their BPMS with a strong affinity towards technological and capability aspects of digital transformation. According to these results, their project is long-term oriented, it leads to building internal capabilities and it encompasses complex technological features that lead to digital transformation in the future. Customer centricity and governance aspects were not neglected but are not in the focus of the project, which means that their BPMS is not the tool for engaging the transformation but for supporting it. Organization A is driven by technology in order to assure digital capabilities for future transformations and could be evaluated as level 2-Managed according to Industry 4.0 MM, and on the conceptual level in relation to DMM [27].

Companies in the operating industry of Organization $\mathrm{B}$ are traditionally strongly dependent on technologies at the operational level. In order to achieve better business results, their business improvement initiatives are therefore driven by other aspects. Resource management, internal process capacity, integration capability, internal efficiency, improvement goals related to quality, time to market, service flexibility and innovation capacity building were the driving factors in organization B to digitally transform itself. This organization is relying on a BPMS that supports these efforts while acting as a mediator towards governance and customer management systems. Due to its industry and its technologically advanced infrastructure, it could be evaluated as level 2-Managed according to Industry 4.0 MM, and on the "transformation defined" level in relation to DMM.

Most important drivers of change for the Organization $\mathrm{C}$ are the governance and the capability aspects since its operating industry relies heavily on standardization and vertical integration. Strategy, mission, vision, goal cascading through the organizational and management levels, operating business rules, building ecosystems, internal process capacity, internal efficiency and service flexibility form the basis of their digital transformation. Customer centricity was not a significant driver in this case, since the scope of their project was related to improving internal operational processes. Overall, this case can be evaluated as an internal transformation focused on building capacities for better reacting to digital disruptions. Organization $\mathrm{C}$ is driven by standardization and vertical integration and could be evaluated as a crossing organization from level 2-Managed to level 3-Established according to Industry 4.0 MM, and on the conceptual level in relation to DMM.

\section{CONCLUSIONS}

In this article we explored the role of BPM Software in the context of digital transformation. First, we investigated what drives companies to transform digitally according to published reports and scientific articles. Four drivers were recognized: Technology-oriented DT, Customer-driven DT, Governance-led DT and Capability-based DT. Since BPM plays an important role in DT initiatives, we mapped technological dimensions of BPMS to DT drivers.

Based on this mapping we proposed a framework to assess the priorities between technological categories of BPMS adoption. Next, we demonstrated how our framework can be used to determine whether these priorities enable digital transformation. The framework relies on the use of the AHP method. We tested the framework on a sample of three organizations and a relatively small number of respondents, which can be seen as a limitation factor. As an addition to our "snowball" literature search, further research could implement other research methods to provide a systematic overview of the literature on the role of BPM in digital transformation. In that way, better insights about the extent and approaches to mapping BPM dimensions into the DT drivers could be gained. Also, in further research the framework could be tested on more cases and by including more employees within the organization. The AHP was selected because of the intention to use a clear method rather than to infer only on the basis of descriptions and qualitative analysis.

After testing the framework for self-assessment on multiple case-studies, priorities based on BPMS features show that: a) some BPMSs are tools for supporting future digital transformation and not for engaging digital change, b) some BPMSs can be seen as middleware between the technological systems in the operational level and highlevel business management systems, and c) some BPMSs are used only for analysis and continuous process optimization while other software or tools are used to achieve digital transformation.

\section{Acknowledgements}

This work has been fully supported by the Croatian Science Foundation under the project PROSPER - Process and Business Intelligence for Business Performance (IP2014-09-3729). 


\section{REFERENCES}

[1] Brynjolfsson, E. \& McAfe, A. (2016). The Second Machine Age: Work, Progress, and Prosperity in a Time of Brilliant Technologies Paperback. W. W. Norton \& Company.

[2] Westerman, G., Bonnet, D., \& McAfee, A. (2014). Leading Digital - turning technology into business transformation. USA: Harvard business review press.

[3] Andriole, S. J. (2018). Skills and Competencies for Digital Transformation. IT Professional, 20(6), 78-81. https://doi.org/10.1109/MITP.2018.2876926

[4] Engels, G., Strothmann, T., \& Teetz, A. (2018). Adapt Cases 4 BPM - A Modeling Framework for Process Flexibility in IIoT. 018 IEEE 22 $2^{\text {nd }}$ International Enterprise Distributed Object Computing Workshop (EDOCW) / Stockholm, 59-68. https://doi.org/10.1109/EDOCW.2018.00020

[5] Bollard, A., Larrea, E., Singla, A., \& Sood, R. (2017). The next-generation operating model for the digital world. Retrieved from https://www.mckinsey.com/businessfunctions/digital-mckinsey/our-insights/the-nextgeneration-operating-model-for-the-digital-world

[6] See TM forum. (2018). Digital Maturity Model Retrieved from https://www.tmforum.org/digital-maturity-modelmetrics/

[7] Newman, D. (2018). Understanding the Six Pillars of Digital Transformation beyond Tech. Retrieved from https://www.forbes.com/sites/danielnewman/2018/05/21/un derstanding-the-six-pillars-of-digital-transformationbeyond-tech/\#5b5c3b2d3f3b

[8] See Forrester. (2018). The Digital Maturity Model 5.0. Retrieved from https://hootsuite.com/resources/forresterthe-digital-maturity-model-5-0\#

[9] von Leipzig, T., Gamp, M., Manz, D., Schöttle, K., Ohlhausen, P., Oosthuizen, G., Palm, D. \& von Leipzig, K. (2017). Initialising Customer-orientated Digital Transformation in Enterprises. Procedia Manufacturing, 8, 517-524. https://doi.org/10.1016/.jpromfg.2017.02.066

[10] Lu, Y. (2017). Industry 4.0: A survey on technologies, applications and open research issues. Journal of Industrial Information Integration, 6, 1-10. https://doi.org/10.1016/j.jii.2017.04.005

[11] Schwab, K. (2017). The fourth industrial revolution. Portfolio penguin: UK.

[12] Osterwalder, Y., Pigneur, G. B., \& Smith, A. (2014). Value proposition design. Hoboken, New Yersey: John Wiley \& Sons.

[13] Evans, N. (2017). Mastering Digital Business: How powerful combinations of disruptive technologies are enabling the next wave of digital transformation. British Computer Society (BSC), UK.

[14] Ismagilova, E., Hughes, L., Dwivedi, Y. K., \& Ravi, R. K. (2019). Smart cities: Advances in research-An information systems perspective. International Journal of Information Management, 47, 88-100. https://doi.org/10.1016/j.jijinfomgt.2019.01.004

[15] See Government of South Australia, (2015). Digital Maturity Assessment Tool - Governance and leadership. Retrieved from

https://digital.sa.gov.au/sites/default/files/content_files/tool kits/Digital_Maturity_Assessment.pdf

[16] Bosilj, V. V., Brkić, Lj., \& Tomičić-Pupek, K. (2018). Understanding the Success Factors in Adopting Business Process Management Software: Case Studies. Interdisciplinary Description of Complex Systems, 16(2), 194-215. https://doi.org/10.7906/indecs.16.2.1

[17] Yin, R. K. (2014). Case study research: design and methods. SAGE Publications, Inc.

[18] Guimarães, M. C., Leal, J. E., \& Mendes, P. (2018). Discrete-event simulation software selection for manufacturing based on the maturity model. Computers in Industry, 103, 14-27.

https://doi.org/10.1016/j.compind.2018.09.005

[19] Bentes, A. V., Carneiro, J., Ferreira da Silva, J., \& Kimura, H. (2012). Multidimensional assessment of organizational performance: Integrating BSC and AHP. Journal of Business Research, 65(12), 1790-1799. https://doi.org/10.1016/j.jbusres.2011.10.039

[20] Saaty, T. L. (2000). Fundamentals of decision making and priority theory with the analytic hierarchy process. RWS Publications, Pittsburgh. https://doi.org/10.1007/978-94-015-9799-9_2

[21] Russo, R. D. F. S. M. \& Camanho, R. (2015). Criteria in AHP: A Systematic Review of Literature. Procedia Computer Science, 55, 1123-1132. https://doi.org/10.1016/j.procs.2015.07.081

[22] Buh, B., Kovačič, A., \& Indihar, Š. M. (2015). Critical success factors for different stages of business process management adoption - a case study. Economic Research Ekonomska istraživanja, 28(1), 243-258. https://doi.org/10.1080/1331677X.2015.1041776

[23] Rosemann, M. (2010). The service portfolio of a BPM center of excellence. Handbook on business process management 1: Introduction, methods and information systems, Springer, Berlin. https://doi.org/10.1007/978-3-642-01982-1_13

[24] Pihir, I., Tomičić-Pupek, K., \& Tomičić, F. M. (2018). Digital Transformation Insights and Trends, Proceedings of the $29^{\text {th }}$ Central European Conference on Information and Intelligent Systems, Varaždin, 141-149.

[25] Asprion, P. M., Schneider, B., \& Grimberg, F. (2018). ERP systems towards digital transformation. Studies in Systems, Decision and Control, 141, 15- 29. https://doi.org/10.1007/978-3-319-74322-6_2

[26] Gökalp, E., Şener, U., \& Eren, P. E. (2017). Development of an Assessment Model for Industry 4.0: Industry 4.0-MM. In: Mas A., Mesquida A., O'Connor R., Rout T., Dorling A. (eds) Software Process Improvement and Capability Determination. SPICE 2017. Communications in Computer and Information Science, 770. Springer, Cham. https://doi.org/10.1007/978-3-319-67383-7_10

[27] Azhari, P., Faraby, N., Rossmann, A., Steimel, B., \& Wichmann, K. S. (2014). Digital transformation report. Neuland GmbH \& Co. KG., Köln.

\section{Contact information:}

Ljiljana BRKIĆ,

Faculty of Electrical Engineering and Computing,

Unska 3, 10000 Zagreb, Croatia

E-mail: ljiljana.brkic@fer.hr

Katarina TOMIČIĆ PUPEK,

Faculty of Organization and Informatics,

Pavlinska 242000 Varaždin, Croatia

E-mail: katarina.tomicic@foi.hr

\section{Vesna BOSILJ VUKŠIĆ,}

(Corresponding author)

Faculty of Economics and Business, University of Zagreb,

Trg J. F. Kennedy 6, 10000 Zagreb, Croatia

E-Mail: vbosilj@efzg.hr 